\title{
IDC Hertzeliya
}

\section{Rephael Recanati International School}

\author{
Seminar Paper
}

Advanced Topics in Social Network Analysis

The Relation between Popularity and Engagement on Facebook

Submitted by:

Giulio Calvosa

Ron Ruzal

$1 / 9 / 2017$ 


\section{Table of contents}

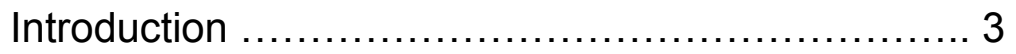

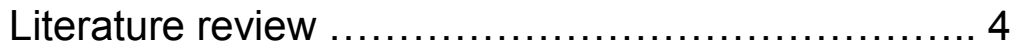

Research Question and Hypothesis ................ 6

Methodology ................................... 7

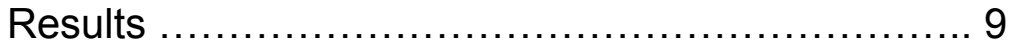

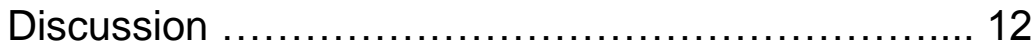

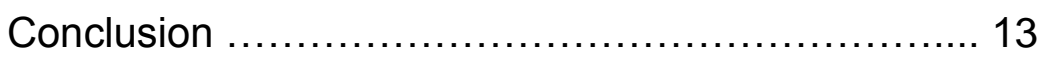

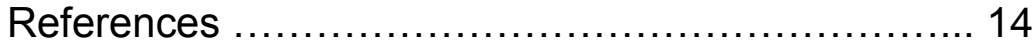




\section{Introduction}

In this paper, we will attempt to find factors that encourage content contribution and crowdsourcing on Facebook groups. This platform for information for various topics is very much based on the factor of group members helping and contributing their own knowledge, information and experiences with others, usually strangers in the real life. We know there is the 99:1 rule that many suggest applies for online content contribution, and we can see this in the large majority of Facebook groups as well as any other platform, when a small portion of a given sample of people is the main contributor to the community. However, in some instances we see lurkers start to participate on content contribution. It can be a one-time participation or a gradual move to be a regular contributor. We will try to examine just that and see what are the factors that influence this behavior online.

This understanding can lead to the interpretation of future behavior and prediction of it, leading to more productive conversations for users who seek information in groups. We can also hypothesis that those factors can be used on a larger scale by brand pages for user engagement on marketing campaigns. 


\section{Literature Review}

Surprisingly at this point of time, when social media websites and applications are such an integrated part of everyday life (Hayat \& Samuel-Azran, 2017) and a common platform for both users and businesses to share content and communicate (Hayat, Samuel-Azran, \& Galily, 2016), we have found relatively little relevant research that deals with the topics of understanding motivators for sharing content and the understanding of the behavior of lurkers.

Little research focuses on lurking in the online environment or considers lurking as a valid and important form of online behavior. This may be due to the fact that there are a number of definitions, and most of them focus on a lack of participation or contribution or see it as a problematic behavior that needs to be changed. Such definitions have given lurkers a negative connotation. They need to be considered as an important factor in online research, starting with a clearer and more positive definition of lurking. It is also necessary to understanding why users decide to lurk, what activities lurkers engage in, and whether the online environment is more valuable by turning lurkers into posters, Edelmann (2013).

In a study conducted by Burke et al. (2009) there was an attempt to try and understand a topic similar to ours. Social networking sites (SNS) are only as good as the content their users share. Therefore, designers of SNS seek to improve the overall user experience by encouraging members to contribute more content. However, user motivations for contribution in SNS are not well understood. This is particularly true for newcomers, who may not recognize the value of contribution - and may even leave the site shortly after registering to it (Hayat, Lesser \& Samuel-Azran, 2017). Using server log data from approximately 140,000 newcomers in Facebook, they predict long-term sharing based on the experiences the newcomers have in their first two weeks. In particular, they find support for social learning: newcomers who see their friends contributing go on to share more content themselves. For newcomers who are initially inclined to contribute, receiving feedback and having a wide audience are also predictors of increased sharing. Burke et al. (2009) conclude the paper with design implications for motivating newcomer sharing in online communities. 
In another research by $\mathrm{Na}$ Sun et al. (2014) they have tried to map the lurkers in online environments and to understand the patterns of their behavior.

In internet culture, lurkers are a special group of website users who regularly login to online communities but seldom post. Their study aims to provide an overall understanding of lurkers by explaining the definition of lurkers, discussing the reasons for lurking and providing suggestions on de-lurking. To understand the reason for lurking, the study first explains why people participate in online communities by building an integrated model of motivational factors of online behaviors. This model classifies motivational factors into four categories: the nature of the online community, individual characteristics, the degree of commitment and quality requirement. Based on this model, four types of lurking reasons are identified: environmental influence, personal preference, individual-group relationship and security consideration. Finally, several strategies for motivating participation in online communities are provided, including external stimuli, improved user-friendliness, encouragement of participation and guidance for newcomers.

In all these studies we did not find a connection between the amount of friends or connection on SNS to the level of activity and participation. In our own study, we will attempt and analyze patterns in our selected online community and see if we identify motivators for sharing content as well as trying to map the users who contribute in the community as well as the lurkers. Leading to our research questions: what makes a group post attract engagement from other users? And, what is the distribution of lurkers and engaged users in the selected group? We also want to examine whether there is a relationship between amount of friends one has on the network and his likelihood of sharing. 


\section{Research Question and Hypothesis}

As mentioned, in all the previous studies we have looked into, we did not find a study that looks into the link between the amount of friends or connections on SNS, and specifically Facebook, to the level of activity and participation. In our own study, we will attempt and analyze patterns in our selected online community and see if we identify motivators for sharing content as well as trying to map the users who contribute in the community as well as the lurkers. Leading to our research questions: what makes a group post attract engagement from other users? and, what is the distribution of lurkers and engaged users in the selected group? We also want to examine our main question, whether there is a relationship between amount of friends one has on the network and his likelihood of sharing content or commenting on other posts.

The argument of this paper suggests that the engagement of a Facebook user on a certain group is directly correlated to his number of friends on the social network. The assumption is that the higher is the engagement of a user on a group, the higher will be his number of friends. This assumption is based on the prior researches that showed the high engagement of social network users affected by various factors, but not the amount of friends they have on the network.

In other previous research conducted on newcomers to Facebook, it was found that social learning had a big part in the predictive factor of these new users to share content. The more they saw their friends share content, the more they were likely to do the same.

Other studies we have found, place much more emphasis on the lurkers perspective of online behavior. This is also an important factor which we will attempt to analyze and see what are the distribution of engaged users and lurkers in our selected group of study, to better help us understand the whole base of users and their patterns of behavior in the group. 


\section{Methodology}

In order to conduct the research and attempt to validate the hypothesis, the methodology of the experiment was divided between a mechanical and a manual analysis of the Facebook group analytics results from the website grytics.com. The website works as a tool for Facebook groups analytics and is able to rank user engagement, number of "likes", comments, posts and more. The Facebook group used for the research is the biggest group of non-Israelis in Israel and it is called "Secret Tel Aviv". We have chose to focus on this group as it gives us a highly diverse range of users, both in gender and age, and ethnicity and country of origin, thus making a very random sample and one that would give us better indication on our research goals. The number of members of the group today is over 78 thousand, but the sample used for the research was the maximum allowed by the free version of the website grytics.com: 5.652 users. The results of the analysis of the website provided a ranking of the most active members of the group according to their engagement on the group on the period, a period between July 20th and August $1^{\text {st }} 2015$.

The website uses an engagement formula that is calculated by the number of comments made by the user $+2 x$ number of the user's posts on the group + the number of content that the user "liked" on the group.

Grytics ranked the users according to their engagement formula and parallely generated a list where it ranked the members of the group with the higher number of friends on their personal networks. After these two lists were generated using the sample of 5.652 group members, the two lists were manually compared, in order to check where the most "popular" group members (the members with the higher number of friends) where placed on the engagement ranking. It should be taken in consideration that, according to the analysis of the website, only less than $1 \%$ of the users create new posts, less than $3 \%$ comment on posts and about only less than $12 \%$ like posts and comments by other users on the group, so the sample used to compare the two ranking lists dropped from 5.652 to a sample of organic engaging members of 822 . 
Therefore, the methodology used on the research was to locate these 822 active group members on the "popularity" ranking, thus expecting to find and accept the hypothesis that as the number of friends on a group member's network grows, also his engagement and will to participate on the group with be higher. 


\section{Results}

The first result we have viewed was the distribution of activity in the selected time period in the group. Resulting in 6\%-posts, $72 \%$-Likes, $22 \%$-comments. As presented in the graph below.

\section{GROUP ACTIVITY}

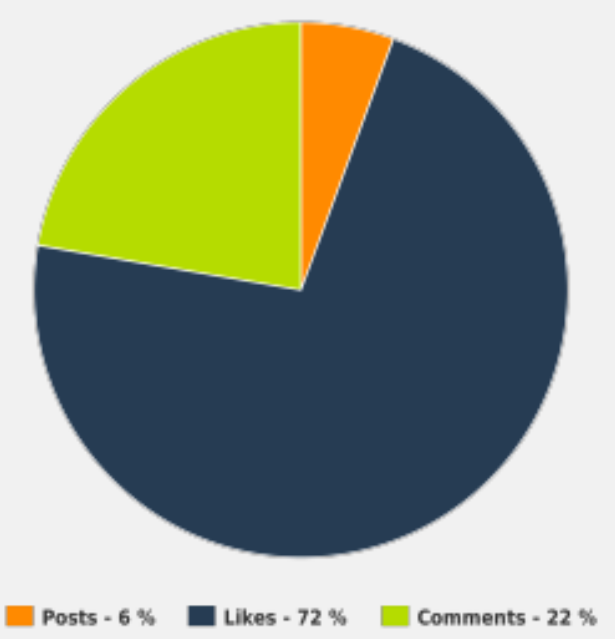

Other information we were able to see was the type of posts that were made during this period, showing the distribution of all types of posts made, as presented below.

\section{POSTS TYPES}

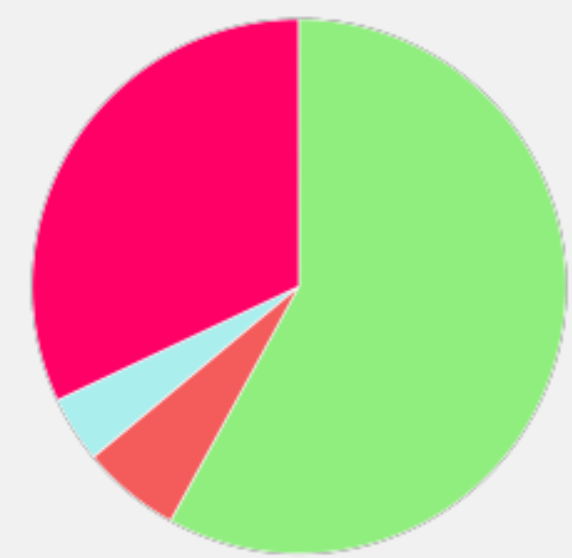

$\square$ Status - $58 \% \square$ Link- $6 \% \square$ Video $-0 \% \square$ Event $-4 \% \square$ photo $-32 \%$ 
We have then went to look at some results focusing more on the members of the group, and looked into the engagement level of the group. This result is based on a random sample of 5652 group members, out of the $78 \mathrm{~K}$ in total. We found that $99.22 \%$ of members are never posting. $97.7 \%$ of members are never commenting on other posts, and $88.8 \%$ of members never Like other posts or comments.

Out of these small percentages of active users, we have targeted down the most active ones. Both in their activity of posting and their activity of commenting on other posts.

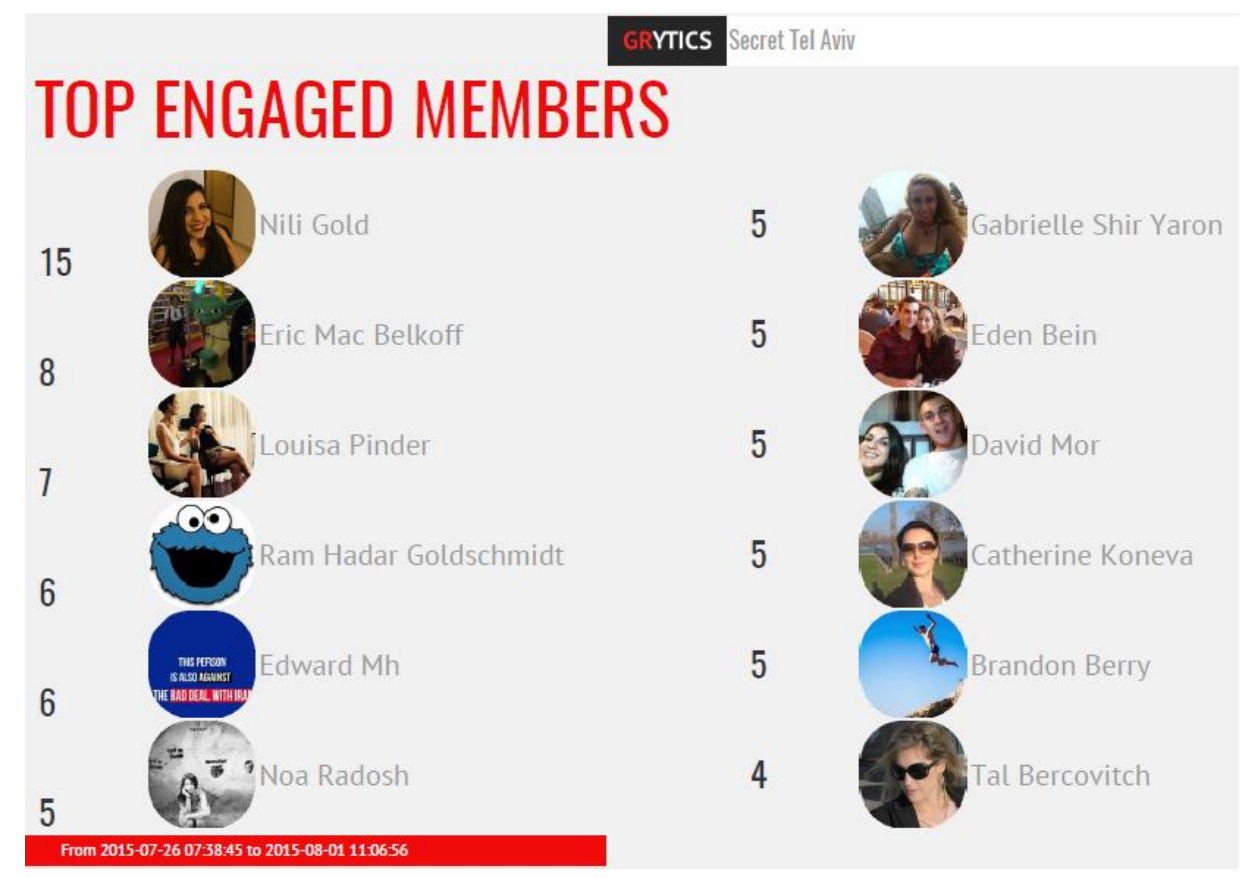

\section{TOP COMMENTERS}

12

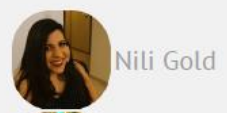

7

6

4

4

4

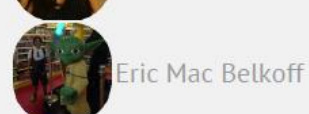

Edward Mh
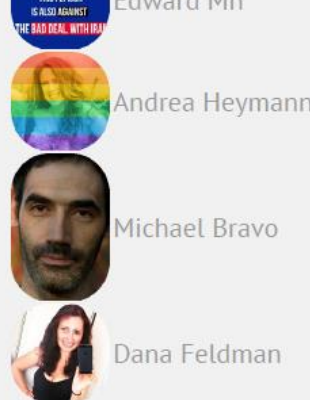

Michael Bravo

Dana Feldman
4

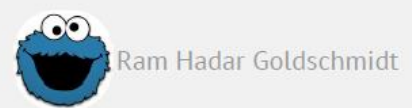

4

4

3

3

3
Nancy Domingo Khemthong

Avi Dorfman 
The last information we needed was a manual comparison of the amount of friends each one of the top most active users in the group has.

We analyzed the 12 most active users presented in the graphs above, as well as 18 more users of each group, to make it 30 members of the top active commenters and 30 top members who were engaged in posting.

In both top 30 members groups, we have found very similar results.

\begin{tabular}{|c|c|c|c|c|}
\hline $\begin{array}{c}\text { Number of } \\
\text { friends }\end{array}$ & $1500+$ & $1200-1500$ & $1000-1200$ & $1000-$ \\
\hline Top Engaged & 5 & 10 & 7 & 8 \\
\hline $\begin{array}{c}\text { Top } \\
\text { Commenters }\end{array}$ & 4 & 12 & 9 & 5 \\
\hline
\end{tabular}

In the top 30 most engaged users, we found $73.33 \%$ to have more than 1000 friends on Facebook. In the top commenters, we have found $83.33 \%$ to have more than 1000 friends.

In addition, $50 \%$ of the top engaged had more than 1200 friends, while $53.33 \%$ of the top commenters had also more than 1200 friends as well. 


\section{Discussion}

The first and foremost finding that was also the most apparent was the sheer amount of non-active users, or lurkers. As they consist of more than $99 \%$ of the sample, it is always fascinating to see how a very tiny portion of the community is actually generation the majority of the content and information. We were expecting this type of finding but were surprised for these extreme percentages in such a relatively small sample. The other finding we saw was the high amount of Facebook friends among the top active users we have analyzed. Our benchmark was one thousand friends for this analysis, and as it turned out, the majority of active users also had a very high number of friends. We cannot draw any conclusion of cause and effect by this finding, but we can definitely see a dominant pattern of one of the characteristics of the most active users in a selected group.

In future research we suggest naturally to expand the Facebook groups on which the study is being conducted to more than one group. Another interesting variable can be the size of the group. As our results are based on a sample of about five thousand out of more than 70 thousand, it would be interesting to see if the same finding will be present in a much smaller group, of no more than one thousand for example. Another possible study can be made in the future on Facebook pages, of businesses and organizations. In such case, the results can potentially be very helpful in marketing strategies and may help generate more engagement with the page, based on the active targeting of paid or non-paid content made by those pages.

One factor that was not mentioned was the fact that in our dividing in the analysis of top Engaged, and top Commenters, there were a few members that fell in both categories; therefore they were treated with no special remarks in the analysis. This perhaps can be done differently. They can be excluded, and a count of the members that are placed in both categories can be made, to see who those 'extra' active ones are. What was also not a topic in the present study is an attempt to analyze the demographics of the most active users, in order to try and make a general profile of characteristics they may have in common. 


\section{Conclusion}

In this research we wanted to better understand the patterns and some characteristics of people who share content online and are actively participation in conversation in Facebook groups. We focused on the group Secret Tel Aviv and found some evidence

that the most active users in the group were also ones that have a lot of friends on the network.

This will hopefully make some contribution to better understanding the behaviors in such groups and will bring new ideas of research in the field of online social networks analysis. 


\section{References}

Burke, M., Marlow, C., \& Lento, T. (2009). Feed me: motivating newcomer contribution in social network sites. Proceedings Of the 27th International Conference on Human Factors in Computing Systems - CHI 09.

Edelmann, N. (2013). Reviewing the Definitions of "Lurkers" and Some Implications for Online Research. Cyberpsychology, Behavior, And Social Networking, 16(9), 645-649.

Grytics.com - Facebook Group Analytics.

Hayat, T. Z., Lesser, O., \& Samuel-Azran, T. (2017). Gendered discourse patterns on online social networks: A social network analysis perspective. Computers in Human Behavior, 77, 132-139.

Hayat, T., \& Samuel-Azran, T. (2017). "You too, Second Screeners?" Second Screeners' Echo Chambers During the 2016 US Elections Primaries. Journal of Broadcasting \& Electronic Media, 61(2), 291-308.

Hayat, T., Samuel-Azran, T., \& Galily, Y. (2016). Al-Jazeera Sport's US Twitter followers: Sport-politics nexus?. Online Information Review, 40(6), 785-797.

Sun, N., Rau, P. P.-L., \& Ma, L. (2014). Understanding lurkers in online communities: A literature review. Computers In Human Behavior, 38, 110-117. 